\title{
Research on a Bilateral Filtering Based Image Denoising Algorithm for Nightly Infrared Monitoring Images
}

\author{
WANG Zhong-ce \\ Jilin Agriculture Science and Technology College \\ Department of Electrical and Information Engineering \\ Jilin, China \\ e-mail:wzcml@163.com
}

\author{
XU Wei \\ Jilin Agriculture Science and Technology College \\ Department of Electrical and Information Engineering \\ Jilin, China \\ e-mail:xuwei_jl@126.com
}

\begin{abstract}
Special information search from video images is a field with very utility value. In this paper, we use the bilateral filter to denoise nightly infrared monitoring images for preprocessing step, so that noises are reduced and edges are preserved. Considering that traditional bilateral filters do not balance grayscale difference and the geometric distance in determining the weights of convolution kernel coefficients, we propose to give the two different values different weights, both of them are fully considered. Experimental results show that this algorithm can effectively remove noise as well as perfectly retain edge information.
\end{abstract}

Keywords-Infrared Image; Denoising; Bilateral Filter; Weighted

\section{INTRODUCTION}

As the infrared imaging keeps on development, this technology has been widely used in civilian, military and other fields [1]. Infrared images are quite different from ordinary natural images in terms of imaging mechanism. Infrared images are also known as thermal images, whose level of darkness reflect temperature of each part of the object and differences emissivity. So it can show the characteristics of the scene. And this feature makes it achieve obvious advantages in the monitor at night. However, the infrared images are disturbed by blurry, noisy and other shortcomings; therefore, the de-noising algorithm for infrared technology has important significance [2]

Image de-noising is an important issue in the field of image processing. It is an important foundation for the other image processing, such as image recognition, understanding. In accordance with processing domains of the image denoising, the existing algorithms can be divided into two categories: spatial domain ones and transform domain ones [3] The former ones refer to those who process image pixels directly. While, the later ones need to map the images that are to de-noised to the transform domain, and process them according to the nature of their transform domain coefficients, and finally transform them into space domain. Spatial domain methods include linear spatial filtering and nonlinear spatial filtering. Linear spatial filtering method is also known as mean filtering, which reduce noise in the image through linear filtering. Nevertheless, it will bring in blurry, and the level of blurring is proportional to the extent of the neighborhood radius [4]. Median filter is one of nonlinear spatial filters, and its basic idea is to use middle pixel gray values in the neighborhood instead of the pixel gray values. This algorithm can reduce the level of noise in the premise of preserving edges or textures. Frequency domain filtering methods include Fourier method and wavelet method [5,6].

Gaussian filter has been widely used in the early denoising applications, but it tends to remove image details (such as textures and edges) in the process of de-nosing. So the literature [7] proposed the bilateral filter method to denoise images. In this method, the convolution kernel is also related to inter-pixel colors (grays) besides geometric distances. Bilateral filter can effectively solve the problems of the Gaussian filter that we have discussed above. However, we note that the bilateral filter just adds the two factors (geometric distance factor and color difference factor) simply, resulting in that the color factor plays a decisive role, and the distance factor is ignored in most cases. Therefore, we propose to use the theory of weights to weight the two factors in order to achieve equal status of the two factors.

\section{RELATED WORKS}

Image Denoising refers to the procedure of predict original images from noisy images. Assuming that the noise image $I$ can be expressed as

$$
I=\tilde{I}+n
$$

Where, $I$ is the noisy image (or observed image), $\tilde{I}$ is the original image (or true image). So, the process of image denoising can be regarded as a mapping from the noisy image I to the original image $\tilde{I}$.

Gaussian smoothing filter is one of very effective low-pass filters in both of spatial domain and frequency domain. It was widely used in the field of image processing at earlier time. It contains five the important properties:

It satisfy rotational invariance;

The Gaussian function is a single-valued function;

The Fourier transform spectrum of the Gaussian function is a single lobe;

The width of the Gaussian filter (determines the degree of smoothing) is characterized by the parameter $\sigma$, and the relationship between $\sigma$ and the degree of smoothing is very simple;

Because of the separability of Gaussian function, the Gaussian filter with larger size may be effectively realized. 
1D Gaussian distribution with mean of zero is defined as

$$
G(x)=\frac{1}{\sqrt{2 \pi \sigma}} e^{-\frac{x^{2}}{2 \sigma^{2}}}
$$

Where, $\sigma$ is the standard deviation of the distribution. Fig.1-(a) is a 1D Gaussian distribution. 2D Gaussian distribution is defined as

$$
G(x, y)=\frac{1}{2 \pi \sigma^{2}} e^{-\frac{x^{2}+y^{2}}{2 \sigma^{2}}}
$$

Fig.1-(b) shows us a 2D Gaussian distribution with mean of zero.

Gaussian filtering is actually a convolution operation of signal and a Gaussian function. Because the image is stored in discrete pixels, so here we need to construct a kernel function in Gaussian distribution. In practical applications, when we calculate the discrete Gaussian approximation, pixels whose distance with center is greater than $3 \sigma$ can be ignored. Typically, the image processing program only needs to calculate matrix with the size of $(6 \sigma+1) \times(6 \sigma+1)$. Fig. 1 (c) is an ordinary Gaussian kernel.

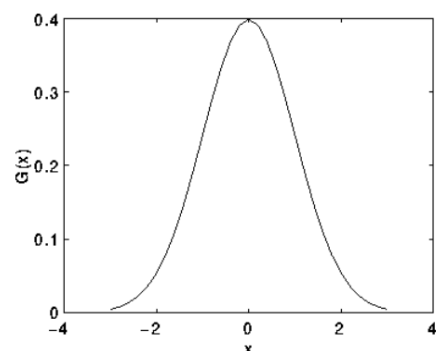

(a)

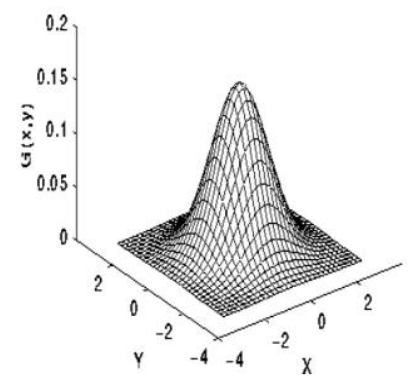

(b)

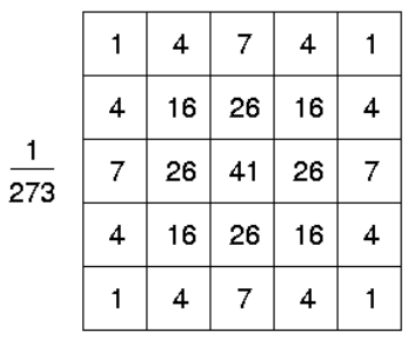

(c)

Figure 1. Gaussian Distribution
Let $I$ denote the image that to be de-noised. $K$ the Gaussian kernel, and $\tilde{I}$ de-noised image,

$$
\tilde{I}=I * K
$$

Where, * is the convolution operation.

\section{DENOISING MODEL}

Bilateral filter was proposed by C. Tomasi was in 1998. Different from traditional image smoothing algorithms (such as Gaussian filter mentioned in section 2), the bilateral filter not only consider pixels geometric closeness, but also takes into account the color differences among neighborhood, which make it more effective in removing noise and preserving edges or textures in images.

Let $I$ denote the noisy image, and $\tilde{I}$ the image after conducting bilateral filtering on $I$. the intensity in image $\tilde{I}$ is defined as,

$$
\tilde{I}(i, j)=\frac{\sum_{k, l} I(k, l) w(i, j, k, l)}{\sum_{k, l} w(i, j, k, l)}
$$

In fact, $w(i, j, k, l)$ can be considered as the weight of $I(k, l)$ in the calculation of the $\tilde{I}(i, j) . w(i, j, k, l)$ is determined by two factors: geometric distance and intensity difference, which are denoted by $d(i, j, k, l)$ and $r(i, j, k, l)$. respectively

$$
w(i, j, k, l)=e^{-(d(i, j, k, l)+r(i, j, k, l))}
$$

Where,

$$
d(i, j, k, l)=e^{-\frac{(i-k)^{2}+(j-l)^{2}}{2 \sigma_{d}^{2}}}
$$

And

$$
r(i, j, k, l)=e^{-\frac{|I(i, j)-I(k, l)|^{2}}{2 \sigma_{d}^{2}}}
$$

However, we note there exist differences between the two ranges; the lower bound of two pixels' distance is 0 , and the upper bound is the size of bilateral filter kernel. While, the range of intensity distance of two pixels is $[0,255]$. Though they are mapped into [0, 1], they still are not balanced. Therefore, this paper proposes a modified form of the Equ.(6).

$$
w(i, j, k, l)=e^{-(\alpha \cdot d(i, j, k, l)+(1-\alpha) \cdot r(i, j, k, l))}
$$

Wherein, $\alpha$ is the weight of $d(i, j, k, l)$, and its range is $[0,1]$. Under normal circumstances, the value is set greater than 0.5 , in order to improve the proportion of $d(i, j, k, l)$ in the calculation of $w(i, j, k, l)$.

\section{EXPERIMENTAL RESULTS AND DISCUSSIONS}

\section{A. Performance Evaluating Measure}

Considering that the purpose of de-noising is to improve image quality, enhance the visual perception, and be conducive to the naked eye, the most reliable method is to 
evaluate the performance of de-noising subjectively. However, the ability of human eye in distinguishing visual difference is limited. In addition, this method can only give qualitative judgment, and it is susceptible to the observation environment, personal preferences. Therefore, in order to assess the performance of de-noising more accurately, the most widely used objective evaluation of quality measure - PSNR (Peak Signal to Noise Ratio) is used in this paper.

PSNR is calculated as

$$
P S N R=10 \log _{10}\left(\frac{255^{2}}{M S E}\right)
$$

Where, MSE is the mean square error between the denoising $\tilde{I}$ and the image reference image $R$, namely

$$
M S E=\frac{\sum_{i=1}^{M} \sum_{j=1}^{N}(\tilde{I}(i, j)-R(i, j))^{2}}{M N}
$$

In effect, $M S E$ measures the difference of image $\tilde{I}$ and $R$.
Different from MSE, A greater PSNR indicates a better denoising image.

\section{B. Performance Evaluating}

In order to verify the effectiveness of the algorithm, we use multiple sets of real infrared images for testing. Here, we list two of them to illustrate the performance of the proposed algorithm. To facilitate the use of objective measure PSNR, we select the infrared image with less noise as a reference image, the test image is obtained by adding white Gaussian noise in the reference image. The comparison results of this algorithm other three de-noising algorithm (mean filtering, Gaussian filtering method and wavelet filtering method) are given. Here we set $\alpha=0.75$.

Fig. 2 shows the first set of test data and denoising experimental results.

If $M S E=0$, it means that $\tilde{I}$ and $R$ are totally the same.

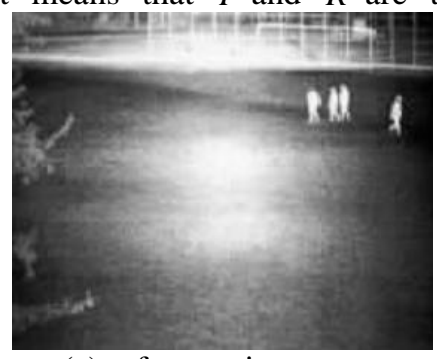

(a) reference image

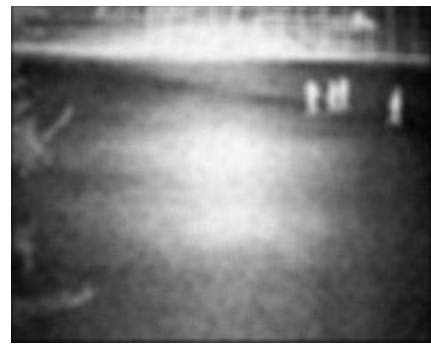

d) Mean filtering

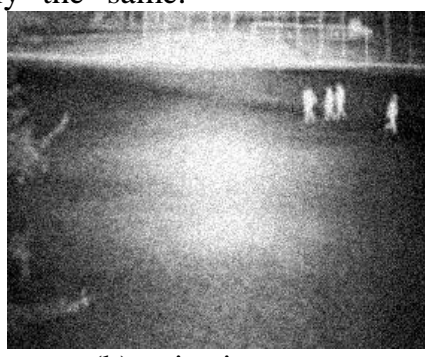

(b) noisy image

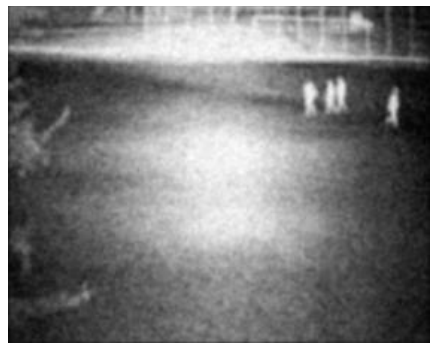

(e) Gaussian smoothing filter

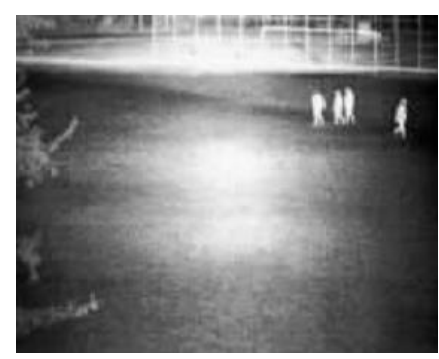

(c) The proposed algorithm

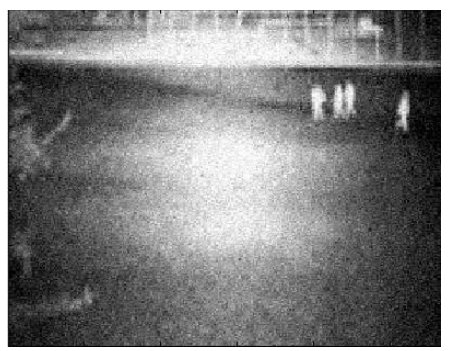

(f) Wavelet De-noising

Figure 2. The first group of images

From Fig. 2, we note that edge blurring phenomenon in the resulting image by mean filter is most obvious, the image by Gaussian filter is better than the image by mean filter, because the Gaussian convolution kernel is in a Gaussian distribution, the weight of the surrounding pixels is small. The wavelet filtering method and the proposed algorithm is better in keeping the edge areas. Table 1 gives an objective assessment of the de-noised images. The table indicates that the proposed algorithm outperform the other three algorithms including wavelet filtering algorithm.
Fig.3 shows another set of experimental data, and the results are similar to the first group. Their objective assessment is also listed in Table 1.

TABLE I. OBJECTIVE EVALUATING RESULTS

\begin{tabular}{|l|c|c|}
\hline \multirow{2}{*}{ Denoising Algorithm } & \multicolumn{2}{|c|}{ PSNR } \\
\cline { 2 - 3 } & Fig.2 & Fig.3 \\
\hline The proposed algorithm & $\mathbf{8 0 . 0 1 2 7}$ & $\mathbf{7 8 . 6 5 9 9}$ \\
\hline Mean filtering & 77.7868 & 75.2363 \\
\hline Gaussian smoothing filter & 79.5049 & 76.3871 \\
\hline Wavelet De-noising & 79.6231 & 75.2782 \\
\hline
\end{tabular}




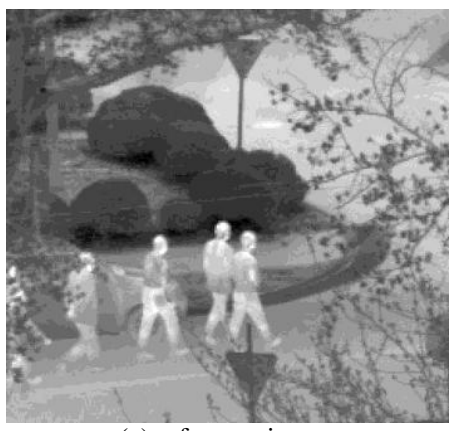

(a) reference image

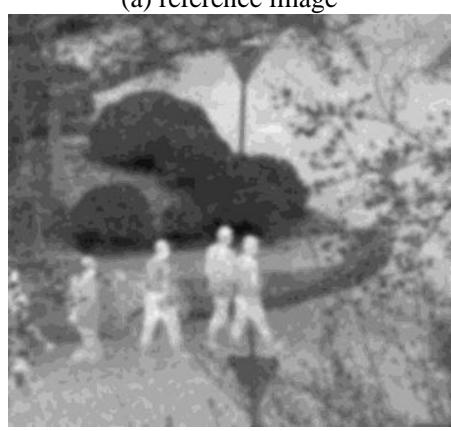

(d) Mean filtering

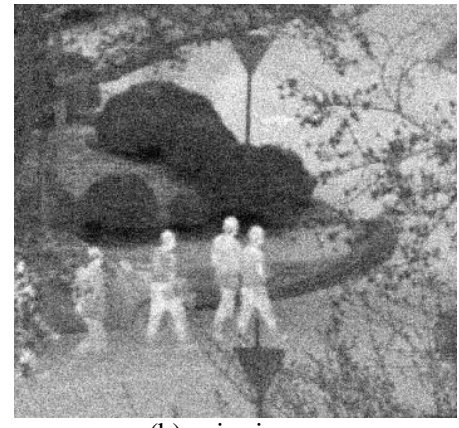

(b) noisy image

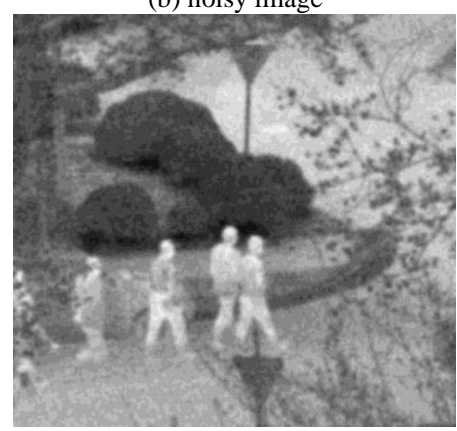

(e) Gaussian smoothing filter

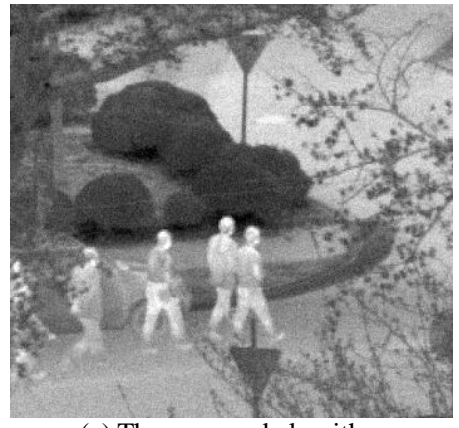

(c) The proposed algorithm

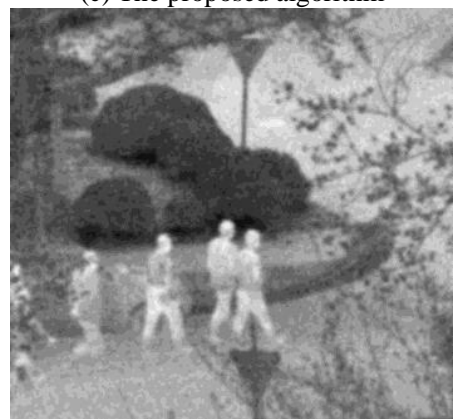

(f) Wavelet De-noising

Figure 3. The second group of images

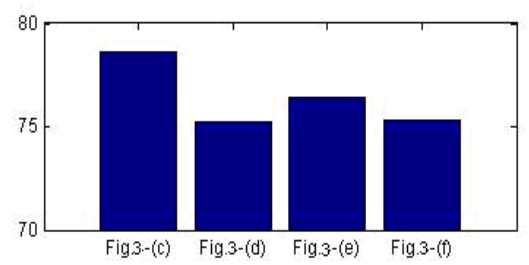

Figure 4. Objective evaluating results of Fig.3

The second set of experimental data is shown in Fig.3, and the results are similar to the first group. Their objective assessment is shown in Fig.4.

From the experimental results, we observed that the proposed algorithm achieves better de-noising effect and it is superior to the other three traditional algorithms in terms of subjective and objective evaluating.

\section{CONCLUSIONS}

For the characteristics infrared images, the bilateral filter is used for filtering them in order to achieve good results that noise are reduced with edges preserved. Considering that traditional bilateral filter does not balance and the gray factor and geometric distance factor, we proposed to give the two factors two different weights in a bid to make them considered equally. Experimental results show that this method can effectively remove noise and preserve edge information well.

\section{ACKNOWLEDGMENT}

We thank the editors and the reviewers for their helpful and constructive suggestions.

\section{REFERENCE}

[1] N. Fujitsuka, H. Naganawa, "Contrast control of far-infrared camera by varying the image sensor temperature", Electronics and Communications in Japan, vol.96, no.7, pp.52-56, 2013.

[2] Smith, L., et al., "Denoising infrared maritime imagery using tailored dictionaries via modified K-SVD algorithm", Applied Optics, vo.51, no.17, pp.3941-3949, 2012.

[3] S Grace Chang, Bin Yu, and Martin Vetterli, "Adaptive wavelet thresholding for image denoising and compression". IEEE Transactions on Image Processing, vol.9,no.9, pp.1532-1546, 2000.

[4] Weifeng Sun, Yuhua Peng. "An Improved Non-Local Means Denoising Approach”. ACTA ELECTRONICA SINICA, vol.38, no.4, pp.923-928, 2010.

[5] Dongfang Jiang, Ming Chen. "A Real-time Wavelet De-noising Algorithm". Chinese Journal of Scientific Instrument. Vol.25, no.6, pp.781-783, 2004

[6] Shiliang Huang, Chaohui Ye, Jianqing Qiu. "Noise Reduction of Magnetic Resonance Images Based on Wavelet Transform: A Review". Chinese Journal of Magnetic Resonance. vol.23, no.4, pp.529-541, 2006.

[7] Tomasi, C., R. Manduchi. "Bilateral filtering for gray and color images". in Sixth International Conference on Computer Vision, 1998. 1998: IEEE. 